\title{
ALMOST AUTOMORPHIC SOLUTIONS TO INTEGRAL EQUATIONS ON THE LINE
}

\author{
CLAUDIO CUEVAS AND CARLOS LIZAMA
}

\begin{abstract}
Given $a \in L^{1}(\mathbb{R})$ and $A$ the generator of an $L^{1}$-integrable family of bounded and linear operators defined on a Banach space $X$, we prove the existence of almost automorphic solution to the semilinear integral equation $u(t)=\int_{-\infty}^{t} a(t-s)[A u(s)+$ $f(s, u(s))] d s$ for each $f: \mathbb{R} \times X \rightarrow X$ almost automorphic in $t$, uniformly in $x \in X$, and satisfying diverse Lipschitz type conditions. In the scalar case, we prove that $a \in L^{1}(\mathbb{R})$ positive, nonincreasing and log-convex is already sufficient.
\end{abstract}

\section{INTRODUCTION}

We study in this paper the almost automorphicity of semilinear integral equations of the form

$$
u(t)=\int_{-\infty}^{t} a(t-s)\left[A u(s)+s^{n} g(s, u(s))\right] d s, \quad t \in \mathbb{R}, n \in \mathbb{Z}_{+}
$$

where $a \in L^{1}(\mathbb{R}), A: D(A) \subset X \rightarrow X$ is the generator of an integral resolvent family defined on a complex Banach space $X$ and $g: \mathbb{R} \times X \rightarrow X$ is an almost automorphic function satisfying suitable Lipschitz conditions.

A continuous function $f: \mathbb{R} \rightarrow X$ is said to be almost automorphic if for every sequence of real numbers $\left(s_{n}^{\prime}\right)_{n \in \mathbb{N}}$ there exists a subsequence $\left(s_{n}\right)_{n \in \mathbb{N}} \subset\left(s_{n}^{\prime}\right)_{n \in \mathbb{N}}$ such that

$$
g(t):=\lim _{n \rightarrow \infty} f\left(t+s_{n}\right)
$$

is well defined for each $t \in \mathbb{R}$, and

$$
f(t)=\lim _{n \rightarrow \infty} g\left(t-s_{n}\right), \quad \text { for each } t \in \mathbb{R} .
$$

Almost automorphicity is a generalization of the classical concept of an almost periodic function. It was introduced in the literature by S. Bochner and recently studied by several authors, including $[4,6,8,10,14,19]$ among others. A complete description of their properties and further applications to evolution equations can be found in the monographs [20] and [21] by G. M. N'Guérékata. Observe that equation (1.1) can be viewed as the limiting equation for the Volterra equation

$$
u(t)=\int_{0}^{t} a(t-s)\left[A u(s)+s^{n} g(s, u(s))\right] d s, \quad t \geq 0, n \in \mathbb{Z}_{+},
$$

2000 Mathematics Subject Classification. Primary 43A60; Secondary 47D60, 45N05, 47J35.

Key words and phrases. Almost automorphic function, integral resolvent family, semilinear integrodifferential equations.

The first author is partially supported by CNPQ/Brazil under Grant 300068/2005-0 .

The second author is partially financed by Laboratorio de Análisis Estocástico, Proyecto Anillo PBCTACT-13. 
see [23, Chapter III, Section 11.5] to obtain details on this assertion.

Sufficient conditions for the existence of almost automorphic solutions of linear and nonlinear evolution equations have been studied in several papers in recent years (see $[19,10,9,14,1,4]$ and $[6])$.

Equation (1.1) arises in the study of heat flow in materials of fading memory type (see [7] and [22]). While the study of the almost automorphic solutions of (1.1) in the particular case $a(t)=t^{\alpha-1} / \Gamma(\alpha)$ with $1 \leq \alpha \leq 2$ was studied in [1], to the knowledge of the authors no results yet exist for the general class of integral equations considered in this paper.

Our plan is as follows: In Section 2, we introduce some preliminaries on integral resolvent families. In section 3 , we treat the linear case

$$
u(t)=\int_{-\infty}^{t} a(t-s)\left[A u(s)+s^{n} f(s)\right] d s, \quad t \in \mathbb{R}, n \in \mathbb{Z}_{+}
$$

and prove our first main result. Section 4 is devoted to the semilinear equation

$$
u(t)=\int_{-\infty}^{t} a(t-s)[A u(s)+f(s, u(s))] d s, \quad t \in \mathbb{R} .
$$

We prove existence of a unique almost automorphic mild solution to (1.4) under the assumption $f$ is almost automorphic and that some Lipchitz condition on $f$ is satisfied.

\section{Preliminaries}

Recall that the Laplace transform of a function $f \in L_{l o c}^{1}\left(\mathbb{R}_{+}, X\right)$ is defined by

$$
\mathcal{L}(f)(\lambda):=\hat{f}(\lambda):=\int_{0}^{\infty} e^{-\lambda t} f(t) d t, \quad \operatorname{Re} \lambda>\omega,
$$

if the integral is absolutely convergent for $\operatorname{Re} \lambda>\omega$. In order to give an operator theoretical approach to equation (1.1) we recall the following definition (cf. [18]).

Definition 2.1. Let $A$ be a closed and linear operator with domain $D(A)$ defined on a Banach space $X$. We call $A$ the generator of an integral resolvent if there exists $\omega \geq 0$ and a strongly continuous function $S: \mathbb{R}_{+} \rightarrow \mathcal{B}(X)$ such that $\{1 / \hat{a}(\lambda): \operatorname{Re} \lambda>\omega\} \subset \rho(A)$ and

$$
\left(\frac{1}{\hat{a}(\lambda)} I-A\right)^{-1} x=\int_{0}^{\infty} e^{-\lambda t} S(t) x d t, \quad \operatorname{Re} \lambda>\omega, \quad x \in X .
$$

In this case, $S(t)$ is called the integral resolvent family generated by $A$.

The concept of integral resolvent, as defined above, is closely related with the concept of resolvent family (see Prüss [23, Chapter I]). A closed but weaker definition was formulated by Prüss [23, definition 1.6]. For the scalar case, where there is a large bibliography, we refer to the monograph by Gripenberg, Londen and Staffans [12], and references therein.

Because of the uniqueness of the Laplace transform, an integral resolvent family with $a(t) \equiv 1$ is the same as a $C_{0}$-semigroup whereas an integral resolvent family with $a(t)=t$ corresponds to the concept of sine family, see [2, Section 3.15].

We note that integral resolvent families are a particular case of $(a, k)$-regularized families introduced in [15]. These are studied in a series of several papers in recent years (see [16], [17], [24]). According to [15] an integral resolvent family $S(t)$ corresponds to a $(a, a)$ regularized family. 
Notably, integral resolvent families are also present in [5, p.62] (see formula (4.33)) in the particular case $a(t)=t^{\alpha-1} / \Gamma(\alpha)$ and where some properties are studied in the context of vector-valued $L^{p}(\mathbb{R}, X)$ spaces.

As in the situation of $C_{0}$-semigroups we have diverse relations of an integral resolvent and its generator. The following result is a direct consequence of [15, Proposition 3.1 and Lemma 2.2].

Proposition 2.2. Let $S(t)$ be an integral resolvent family on $X$ with generator $A$. Then the following holds:

(a) $S(t) D(A) \subset D(A)$ and $A S(t) x=S(t) A x$ for all $x \in D(A), t \geq 0$;

(b) Let $x \in D(A)$ and $t \geq 0$. Then

$$
S(t) x=a(t) x+\int_{0}^{t} a(t-s) A S(s) x d s .
$$

(c) Let $x \in X$ and $t \geq 0$. Then $\int_{0}^{t} a(t-s) S(s) x d s \in D(A)$ and

$$
S(t) x=a(t) x+A \int_{0}^{t} a(t-s) S(s) x d s .
$$

In particular, $S(0)=a(0)$.

If an operator $A$ with domain $D(A)$ is the infinitesimal generator of an integral resolvent family $S(t)$ and $a(t)$ is a continuous, positive and nondecreasing function which satisfies $\varlimsup_{t \rightarrow 0^{+}} \frac{\|S(t)\|}{a(t)}<\infty$, then for all $x \in D(A)$ we have

$$
A x=\lim _{t \rightarrow 0^{+}} \frac{S(t) x-a(t) x}{(a * a)(t)},
$$

see [17, Theorem 2.1]. For example, the case $a(t) \equiv 1$ corresponds to the generator of a $C_{0}$-semigroup and $a(t)=t$ actually corresponds to the generator of a sine family.

A characterization of generators of integral resolvent families, analogous to the HilleYosida Theorem for $C_{0}$ semigroups, can be directly deduced from [15, Theorem 3.4]. Results on perturbation, approximation, representation as well as ergodic type theorems can be also deduced from the more general context of $(a, k)$ regularized resolvents (see $[16,17]$ and [24]).

\section{Almost Automorphic Solutions}

In this section we consider the existence and uniqueness of almost automorphic solutions to the evolution equation

$$
u(t)=\int_{-\infty}^{t} a(t-s)\left[A u(s)+s^{n} g(s)\right] d s, \quad t \in \mathbb{R}, n \in \mathbb{Z}_{+}
$$

where $A$ is the generator of an integral resolvent family and $a \in L^{1}(\mathbb{R})$. 
As a consequence of the definition of an almost automorphic function given in the introduction, the following properties hold (cf [21]) : let $f, g: \mathbb{R} \rightarrow X$ be almost automorphic functions and let $\lambda \in \mathbb{R}$, then $f+g, \lambda f$ and $f_{\lambda}$ are almost automorphic, where $f_{\lambda}(t):=f(t+\lambda)$. Moreover, the range $R(f)$ of $f$ is relatively compact, therefore it is bounded. Almost automorphic functions constitute a Banach space $A A(X)$ when it is endowed with the sup norm:

$$
\|f\|_{\infty}:=\sup _{t \in \mathbb{R}}\|f(t)\| .
$$

The following result on the almost automorphicity of the convolution is the key for the results of this paper. It can be proved by the simple argument of [5, Proposition 2.3]. See also [6, Theorem 2.1] or [1, Lemma 3.1] for a detailed proof.

Lemma 3.1. Let $\{S(t)\}_{t \geq 0} \subset \mathcal{B}(X)$ be a strongly continuous family of bounded and linear operators such that

$$
\|S(t)\| \leq \phi(t) \text { for all } t \in \mathbb{R}_{+} \text {with } \phi \in L^{1}\left(\mathbb{R}_{+}\right) .
$$

If $f: \mathbb{R} \rightarrow X$ is an almost automorphic function then

$$
\int_{-\infty}^{t} S(t-s) f(s) d s \in A A(X) .
$$

Proposition 3.2. Let $a \in L^{1}(\mathbb{R})$. Assume that A generates an integral resolvent family $\{S(t)\}_{t \geq 0}$ on $X$, which is in addition integrable. If $f$ is almost automorphic and takes values on $D(A)$ then the unique bounded solution of the problem

$$
u(t)=\int_{-\infty}^{t} a(t-s)[A u(s)+f(s)] d s, \quad t \in \mathbb{R},
$$

is almost automorphic and is given by

$$
u(t)=\int_{-\infty}^{t} S(t-s) f(s) d s, \quad t \in \mathbb{R} .
$$

Proof. Since $f(t) \in D(A)$ for all $t \in \mathbb{R}$, we obtain $u(t) \in D(A)$ for all $t \in \mathbb{R}$ (see [23, Proposition 1.2]). Then by (2.1) and Fubini's theorem we obtain $\int_{-\infty}^{t} a(t-s) A u(s) d s=$ $u(t)-\int_{-\infty}^{t} a(t-s) f(\tau) d \tau$. The statement follows by Lemma 3.1.

Remark 3.3. A complete discussion on integrable integral resolvents is given in [23, Chapter 3 , section 10].

If $w_{n}(t):=(1+|t|)^{n}, n \in \mathbb{Z}_{+}, t \in \mathbb{R}$ and $f: \mathbb{R} \rightarrow X$ we set

$$
\|f\|_{w_{n}, \infty}:=\left\|f / w_{n}\right\|_{\infty} .
$$

We consider the following weighted classes (see [4])

$$
A A_{w_{n}}(X):=\left\{w_{n} f: f \in A A(X)\right\} \text { and } C_{w_{n}, 0}(\mathbb{R}, X):=\left\{w_{n} f: f \in C_{0}(\mathbb{R} ; X)\right\} .
$$

Then one can check that $A A_{w_{n}}(X)$ and $C_{w_{n}, 0}(\mathbb{R}, X)$ are Banach spaces endowed with the norm $\|\cdot\|_{w_{n}, \infty}$. Moreover, it was proved in [4] that $A A_{w_{n}}(X)+C_{w_{n}, 0}(\mathbb{R}, X)$ is a closed subspace of $B C_{w_{n}}(\mathbb{R}, X)$ and that the sum is (topologically) direct, see [4, Theorem 1.6] and the remark before Definition 3.2 in the cited paper.

The following is the main result of this section. Their proof follows the same lines of $[4$, Theorem 4.3] (see also [1]), but we give it here for the sake of completeness. 
Theorem 3.4. Let $n \in \mathbb{Z}_{+}$. Assume that A generates an integral resolvent family $\{S(t)\}_{t \geq 0}$ satisfying

$$
\left\|t^{k} S(t)\right\| \leq \phi(t), t \in \mathbb{R}_{+}, \text {with } \phi \in L^{1}\left(\mathbb{R}_{+}\right)
$$

for all $k=0,1, \ldots, n$. Let $f \in A A(X)$ taking values on $D(A)$. Then the equation

$$
u(t)=\int_{-\infty}^{t} a(t-s)\left[A u(s)+s^{n} f(s)\right] d s, \quad t \in \mathbb{R},
$$

has a unique solution $u \in A A_{w_{n}}(X) \oplus C_{w_{n}, 0}(\mathbb{R}, X)$.

Proof. Let $u(t)=\int_{0}^{\infty} S(s)(t-s)^{n} f(t-s) d s$ then

$$
\begin{aligned}
\int_{0}^{\infty} S(s)(t-s)^{n} f(t-s) d s & =t^{n} \int_{0}^{\infty} S(s) f(t-s) d s \\
& +\sum_{k=1}^{n}(-1)^{k}\left(\begin{array}{c}
n \\
k
\end{array}\right) t^{n-k} \int_{0}^{\infty} s^{k} S(s) f(t-s) d s \\
=: & u_{1}(t)+u_{2}(t) .
\end{aligned}
$$

(in case $n=0$ we take $\left.u_{2}(t) \equiv 0\right)$. By Lemma 3.1, $u_{1} \in A A_{w_{n}}(X)$. We will show that $u_{2} \in C_{w_{n}, 0}(\mathbb{R}, X)$. Indeed, by $(3.3)$ we have $s^{k} S(\cdot) \in L^{1}\left(\mathbb{R}_{+}, \mathcal{B}(X)\right)$ for all $k=0,1, \ldots, n$. So

$$
\left\|\int_{0}^{\infty} s^{k} S(s) f(t-s) d s\right\| \leq \int_{0}^{\infty}\left\|s^{k} S(s) f(t-s)\right\| d s \leq\|f\|_{\infty}\left\|\phi_{k}\right\|_{1}
$$

for all $k=0,1, \ldots, n$. Since $\lim _{|t| \rightarrow \infty} \frac{t^{r}}{(1+|t|)^{n}}=0$, we have

$$
t^{r} \int_{0}^{\infty} s^{k} S(s) f(t-s) d s \in C_{w_{n}, 0}(\mathbb{R}, X), \quad 0 \leq r<n
$$

and this shows $u_{2} \in C_{w_{n}, 0}(\mathbb{R}, X)$.

Taking $n=0$ and $X=\mathbb{R}$ we obtain the following result for the scalar case.

Corollary 3.5. Let $f: \mathbb{R} \rightarrow \mathbb{R}$ be an almost automorphic function, $a \in L^{1}(\mathbb{R})$ and let $\rho>0$ be a real number. Then the equation

$$
u(t)=\int_{-\infty}^{t} a(t-s)[-\rho u(s)+f(s)] d s, \quad t \in \mathbb{R},
$$

has an almost automorphic solution given by

$$
u(t)=\int_{-\infty}^{t} S_{\rho}(t-s) f(s) d s, \quad t \in \mathbb{R}
$$

whenever $S_{\rho}(t)$, being the solution of the one dimensional equation

$$
S_{\rho}(t)=a(t)-\rho \int_{0}^{t} a(t-s) S_{\rho}(s) d s,
$$

satisfy $\left|S_{\rho}(t)\right| \leq \phi_{\rho}(t)$, with $\phi_{\rho} \in L^{1}\left(\mathbb{R}_{+}\right)$. 
Example 3.6. Consider $a(t)=\frac{t^{\alpha-1}}{\Gamma(\alpha)} e^{-\beta t}$ where $\beta>0$ and $1<\alpha<2$. Then we can check that

$$
S_{\rho}(t)=t^{\alpha-1} E_{\alpha, \alpha}(-\rho t) e^{-\beta t}
$$

where $E_{\alpha, \alpha}$ denotes the generalized Mittag-Leffler function (see e.g. [11]) which is defined by

$$
E_{\alpha, \beta}(z):=\sum_{n=0}^{\infty} \frac{z^{n}}{\Gamma(\alpha n+\beta)} \quad \alpha, \beta>0, \quad z \in \mathbb{C} .
$$

Using the explicit description of $S_{\rho}(t) e^{\beta t}$ given in [1, Corollary 3.7] we can show that $\left|S_{\rho}(t)\right| \leq \phi_{\rho}(t)$, with $\phi_{\rho} \in L^{1}\left(\mathbb{R}_{+}\right)$. We conclude that the equation

$$
u(t)=\int_{-\infty}^{t} \frac{(t-s)^{\alpha-1}}{\Gamma(\alpha)} e^{-\beta(t-s)}[-\rho u(s)+f(s)] d s, \quad t \in \mathbb{R},
$$

has an almost automorphic solution whenever $f$ is almost automorphic.

Notably, the following result provide a wide class of kernels $a(t)$ such that the condition $\left|S_{\rho}(t)\right| \leq \phi_{\rho}(t)$, with $\phi_{\rho} \in L^{1}\left(\mathbb{R}_{+}\right)$of Corollary (3.5) holds.

Corollary 3.7. Let $f: \mathbb{R} \rightarrow \mathbb{R}$ be an almost automorphic function and let $\rho>0$ be a real number. Suppose $a \in L^{1}(\mathbb{R})$ is positive, nonincreasing and log-convex, then

a) There is $S_{\rho} \in L^{1}\left(\mathbb{R}_{+}\right) \cap C\left(\mathbb{R}_{+}\right)$such that equation (3.5) is satisfied;

b) The equation

$$
u(t)=\int_{-\infty}^{t} a(t-s)[-\rho u(s)+f(s)] d s, \quad t \in \mathbb{R},
$$

has a mild almost automorphic solution given by

$$
u(t)=\int_{-\infty}^{t} S_{\rho}(t-s) f(s) d s, \quad t \in \mathbb{R} .
$$

Proof. Part a) follows by [23, Lemma 4.1, p.98] and then part b) is a consequence of the previous corollary.

\section{Semilinear Integral Equations on the Line}

In this section we study the semilinear equation

$$
u(t)=\int_{-\infty}^{t} a(t-s)[A u(s)+f(s, u(s))] d s, \quad t \in \mathbb{R} .
$$

Definition 4.1. Let $A$ be the generator of an integral resolvent family $\{S(t)\}_{t \geq 0}$. A continuous function $u: \mathbb{R} \rightarrow X$ satisfying the integral equation

$$
u(t)=\int_{-\infty}^{t} S(t-s) f(s, u(s)) d s, \text { for all } t \in \mathbb{R},
$$

is called a mild solution on $\mathbb{R}$ to the equation (4.1). 
Theorem 4.2. Assume that $A$ generates an integral resolvent family $\{S(t)\}_{t \geq 0}$ such that

$$
\|S(t)\| \leq \phi(t), \text { for all } t \geq 0 \text {, with } \phi \in L^{1}\left(\mathbb{R}_{+}\right) .
$$

Let $f: \mathbb{R} \times X \rightarrow X$ be almost automorphic in $t$ uniformly in $x \in X$ and satisfy a Lipschitz condition in $x$ uniformly in $t$, that is,

$$
\|f(t, x)-f(t, y)\| \leq L\|x-y\|, \text { for all } x, y \in X .
$$

Then

$$
u(t)=\int_{-\infty}^{t} a(t-s)[A u(s)+f(s, u(s))] d s, \quad t \in \mathbb{R},
$$

has a unique almost automorphic mild solution whenever $L<\|\phi\|_{1}^{-1}$.

Proof. We define the operator $F: A A(X) \mapsto A A(X)$ by

$$
(F \varphi)(t):=\int_{-\infty}^{t} S(t-s) f(s, \varphi(s)) d s, \quad t \in \mathbb{R} .
$$

In view of [13, Lemma 2.2] (see also [21]) and Lemma 3.1, $F$ is well defined. Then for $\varphi_{1}, \varphi_{2} \in A A(X)$ we have:

$$
\begin{aligned}
\left\|F \varphi_{1}-F \varphi_{2}\right\|_{\infty} & =\sup _{t \in \mathbb{R}}\left\|\int_{-\infty}^{t} S(t-s)\left[f\left(s, \varphi_{1}(s)\right)-f\left(s, \varphi_{2}(s)\right)\right] d s\right\| \\
& \leq L \sup _{t \in \mathbb{R}} \int_{0}^{\infty}\|S(\tau)\|\left\|\varphi_{1}(t-\tau)-\varphi_{2}(t-\tau)\right\| d \tau \\
& \leq L\left\|\varphi_{1}-\varphi_{2}\right\|_{\infty} \int_{0}^{\infty} \phi(\tau) d \tau .
\end{aligned}
$$

This proves that $F$ is a contraction, so by the Banach fixed point theorem there exists a unique $u \in A A(X)$, such that $F u=u$, that is $u(t)=\int_{-\infty}^{t} S(t-s) f(s, u(s)) d s$.

An immediate consequence of Theorem 4.2 and Corollary 3.7 is the following remarkable result.

Corollary 4.3. Let $\rho>0$ be a real number. Suppose $a \in L^{1}(\mathbb{R})$ is positive, nonincreasing and log-convex and let $f: \mathbb{R} \times \mathbb{R} \rightarrow \mathbb{R}$ be almost automorphic in the first variable uniformly with respect to the second variable, and satisfies a Lipschitz condition in the second variable, that is,

$$
\|f(t, x)-f(t, y)\| \leq L\|x-y\|, \text { for all } x, y \in \mathbb{R} .
$$

Then there is $S_{\rho} \in L^{1}\left(\mathbb{R}_{+}\right) \cap C\left(\mathbb{R}_{+}\right)$satisfying the linear equation (3.5). Moreover, the semilinear equation

$$
u(t)=\int_{-\infty}^{t} a(t-s)[-\rho u(s)+f(s, u(s))] d s, \quad t \in \mathbb{R},
$$

has a unique almost automorphic mild solution whenever $L<\left\|S_{\rho}\right\|_{1}^{-1}$.

A different Lipschitz condition is provided in the following result. 
Theorem 4.4. Assume that $A$ generates an integral resolvent family $\{S(t)\}_{t \geq 0}$ such that

$$
\|S(t)\| \leq \phi(t), \text { for all } t \geq 0 \text {, with } \phi \in L^{1}\left(\mathbb{R}_{+}\right) .
$$

Let $f: \mathbb{R} \times X \rightarrow X$ be almost automorphic in $t$ uniformly in $x \in X$ and satisfy the Lipschitz condition

$$
\|f(t, x)-f(t, y)\| \leq L(t)\|x-y\|, \text { for all } x, y \in X, t \in \mathbb{R},
$$

where $L \in L^{1}(\mathbb{R})$. Then equation (4.1) has a unique almost automorphic mild solution.

Proof. We define the operator $F$ as in (4.4). Let $\varphi_{1}, \varphi_{2}$ be in $A A(X)$ and denote $C:=$ $\sup _{t \in \mathbb{R}}\|S(t)\|$. We have:

$$
\begin{aligned}
\left\|\left(F \varphi_{1}\right)(t)-\left(F \varphi_{2}\right)(t)\right\| & =\left\|\int_{-\infty}^{t} S(t-s)\left[f\left(s, \varphi_{1}(s)\right)-f\left(s, \varphi_{2}(s)\right)\right] d s\right\| \\
& \leq \int_{-\infty}^{t} L(s)\|S(t-s)\|\left\|\varphi_{1}(s)-\varphi_{2}(s)\right\| d s \\
& \leq C\left\|\varphi_{1}-\varphi_{2}\right\|_{\infty}\|L\|_{1} .
\end{aligned}
$$

In general we get

$$
\begin{aligned}
\left\|\left(F^{n} \varphi_{1}\right)(t)-\left(F^{n} \varphi_{2}\right)(t)\right\| & \leq \frac{C^{n}}{(n-1) !}\left(\int_{-\infty}^{t} L(s)\left(\int_{-\infty}^{s} L(\tau) d \tau\right)^{n-1} d s\right)\left\|\varphi_{1}-\varphi_{2}\right\|_{\infty} \\
& \leq \frac{C^{n}}{n !}\left(\int_{-\infty}^{t} L(\tau) d \tau\right)^{n}\left\|\varphi_{1}-\varphi_{2}\right\|_{\infty} \\
& \leq \frac{\left(C\|L\|_{1}\right)^{n}}{n !}\left\|\varphi_{1}-\varphi_{2}\right\|_{\infty} .
\end{aligned}
$$

Hence, since $\frac{\left(C\|L\|_{1}\right)^{n}}{n !}<1$ for $n$ sufficiently large, by the contraction principle $F$ has a unique fixed point $u \in A A(X)$.

We notice that in (4.5) different type of conditions can be considered for $L(t)$. This fact is studied in the following results.

Theorem 4.5. Assume that $A$ generates an integral resolvent family $\{S(t)\}_{t \geq 0}$ such that

$$
\|S(t)\| \leq \phi(t), \text { for all } t \geq 0, \text { with } \phi \in L^{1}\left(\mathbb{R}_{+}\right) .
$$

Let $f: \mathbb{R} \times X \rightarrow X$ be almost automorphic in $t$ uniformly in $x \in X$ and satisfy the Lipschitz condition

$$
\|f(t, x)-f(t, y)\| \leq L(t)\|x-y\|, \text { for all } x, y \in X, t \in \mathbb{R}
$$

where the integral $\int_{-\infty}^{t} L(s) d s$ exists for all $t \in \mathbb{R}$. Then equation (4.1) has a unique almost automorphic mild solution.

Proof. Define a new norm $\|\varphi\|:=\sup _{t \in \mathbb{R}}\{v(t)\|\varphi(t)\|\}$, where $v(t):=e^{-k \int_{-\infty}^{t} L(s) d s}$ and $k$ is a fixed positive constant greater than $C:=\sup _{t \in \mathbb{R}}\|S(t)\|$. Let $\varphi_{1}, \varphi_{2}$ be in $A A(X)$, 
then we have

$$
\begin{aligned}
v(t)\left\|\left(F \varphi_{1}\right)(t)-\left(F \varphi_{2}\right)(t)\right\| & =v(t)\left\|\int_{-\infty}^{t} S(t-s)\left[f\left(s, \varphi_{1}(s)\right)-f\left(s, \varphi_{2}(s)\right)\right] d s\right\| \\
& \leq C \int_{-\infty}^{t} v(t) L(s)\left\|\varphi_{1}(s)-\varphi_{2}(s)\right\| d s \\
& \leq C \int_{-\infty}^{t} v(t) v(s)^{-1} L(s) v(s)\left\|\varphi_{1}(s)-\varphi_{2}(s)\right\| d s \\
& \leq C\left|\left\|\varphi_{1}-\varphi_{2}\right\|\right| \int_{-\infty}^{t} v(t) v(s)^{-1} L(s) d s \\
& =\frac{C}{k}\left|\left\|\varphi_{1}-\varphi_{2}\right\|\right| \int_{-\infty}^{t} k e^{k \int_{t}^{s} L(\tau) d \tau} L(s) d s \\
& =\frac{C}{k}\left|\left\|\varphi_{1}-\varphi_{2}\right\|\right| \int_{-\infty}^{t} \frac{d}{d s}\left(e^{k \int_{t}^{s} L(\tau) d \tau}\right) d s \\
& =\frac{C}{k}\left[1-e^{-k \int_{-\infty}^{t} L(\tau) d \tau}\right]\left\|\varphi_{1}-\varphi_{2} \mid\right\| \\
& \leq \frac{C}{k} \mid\left\|\varphi_{1}-\varphi_{2}\right\| \| .
\end{aligned}
$$

Hence, since $C / k<1, F$ has a unique fixed point $u \in A A(X)$.

Theorem 4.6. Assume that $A$ generates an integral resolvent family $\{S(t)\}_{t \geq 0}$ such that

$$
\|S(t)\| \leq \phi(t), \text { for all } t \geq 0
$$

where $\phi: \mathbb{R}_{+} \rightarrow \mathbb{R}_{+}$is a decreasing function such that $\phi_{0}:=\sum_{m=0}^{\infty} \phi(m)<\infty$. Let $f: \mathbb{R} \times X \rightarrow X$ almost automorphic in $t$ uniformly in $x \in X$ satisfying the Lipschitz condition

$$
\|f(t, x)-f(t, y)\| \leq L(t)\|x-y\|, \text { for all } x, y \in X, t \in \mathbb{R}
$$

where $L:=\sup _{t \in \mathbb{R}} \int_{t}^{t+1} L(s) d s<\infty$ Then equation (4.1) has a unique almost automorphic mild solution whenever $L \phi_{0}<1$.

Proof. Since $\phi$ is a decreasing function such that $\sum_{m=0}^{\infty} \phi(m)<\infty$ we have that $\phi \in$ $L^{1}\left(\mathbb{R}_{+}\right)$and hence $S(t)$ is integrable. Let $\varphi_{1}, \varphi_{2}$ be in $A A(X)$, then for the same operator 
$F$ defined in (4.4), we have

$$
\begin{aligned}
\left\|\left(F \varphi_{1}\right)(t)-\left(F \varphi_{2}\right)(t)\right\| & =\left\|\int_{-\infty}^{t} S(t-s)\left[f\left(s, \varphi_{1}(s)\right)-f\left(s, \varphi_{2}(s)\right)\right] d s\right\| \\
& \leq \int_{-\infty}^{t} L(s)\|S(t-s)\|\left\|\varphi_{1}(s)-\varphi_{2}(s)\right\| d s \\
& \leq\left(\sum_{m=0}^{\infty} \int_{t-(m+1)}^{t-m} L(s)\|S(t-s)\| d s\right)\left\|\varphi_{1}-\varphi_{2}\right\|_{\infty} \\
& \leq\left(\sum_{m=0}^{\infty} \int_{t-(m+1)}^{t-m} L(s) \phi(t-s) d s\right)\left\|\varphi_{1}-\varphi_{2}\right\|_{\infty} \\
& \leq\left(\sum_{m=0}^{\infty} \phi(m) \int_{t-(m+1)}^{t-m} L(s) d s\right)\left\|\varphi_{1}-\varphi_{2}\right\|_{\infty} \\
& \leq L\left(\sum_{m=0}^{\infty} \phi(m)\right)\left\|\varphi_{1}-\varphi_{2}\right\|_{\infty}=L \phi_{0}\left\|\varphi_{1}-\varphi_{2}\right\|_{\infty},
\end{aligned}
$$

which finish the proof.

\section{REFERENCES}

[1] D. Araya, C. Lizama. Almost automorphic mild solutions to fractional differential equations, Nonlinear Analysis Series A: Theory Methods \& Appl., 69 (11) (2008), 3692-3705.

[2] W. Arendt, C. Batty, M. Hieber, F. Neubrander. Vector-valued Laplace Transforms and Cauchy Problems. Monographs in Mathematics. 96, Birkhäuser, Basel, 2001.

[3] B. Basit. Harmonic Analysis and Asymptotic Behavior of Solutions to the Abstract Cauchy Problem. Semigroup Forum 54, (1997), 58-74.

[4] B. Basit, A.J. Pryde. Asymptotic behavior of orbits of $C_{0}$-semigroups and solutions of linear and semilinear abstract differential equations, Russ. J. Math. Phys. 13 (1) (2006), 13-30.

[5] E. Bazhlekova. Fractional Evolution Equations in Banach Spaces, Ph.D. Thesis, Eindhoven University of Technology, 2001.

[6] D. Bugajewski, T. Diagana. Almost automorphy of the convolution operator and applications to differential and functional differential equations, Nonlinear Stud., 13 (2) (2006), 129-140.

[7] Ph. Clément, G. Da Prato. Existence and regularity results for an integral equation with infinite delay in a Banach space, Integral Equations and Operator Theory, 11 (1988), 480-500.

[8] T. Diagana. Some remarks on some second-order hyperbolic differential equations, Semigroup Forum 68 (2004), 357-364.

[9] T. Diagana, G.M. N'Guérékata. Almost automorphic solutions to semilinear evolution equations, Funct. Differ. Equ. 13 (2) (2006), 195-206.

[10] J.A. Goldstein, G.M.N'Guérékata. Almost automorphic solutions of semilinear evolution equations, Proc. Amer. Math. Soc. 133 (8) (2005), 2401-2408.

[11] R. Gorenflo, F. Mainardi. On Mittag-Leffler-type functions in fractional evolution processes, J. Comp. Appl. Math. 118 (2000), 283-299.

[12] G. Gripenberg, S-O Londen, O. Staffans. Volterra Integral and Functional Equations. Encyclopedia of Mathematics and Applications, 34, Cambridge University Press, Cambridge-New York, 1990.

[13] J. Liang; J. Zhang; T.J. Xiao, Composition of pseudo almost automorphic and asymptotically almost automorphic functions. J. Math. Anal. Appl. 340 (2008), no. 2, 1493-1499.

[14] J. Liu, G.M. N'Guérékata, N. van Minh. Almost automorphic solutions of second order evolution equations, Appl. Anal. 84 (11) (2005), 1173-1184. 
[15] C. Lizama. Regularized solutions for abstract Volterra equations, J. Math. Anal. Appl. 243 (2000), $278-292$.

[16] C. Lizama. On approximation and representation of k-regularized resolvent families, Integral Equations Operator Theory 41 (2), (2001), 223-229.

[17] C. Lizama, J. Sánchez. On perturbation of k-regularized resolvent families, Taiwanese J. Math. 7 (2), (2003), 217-227.

[18] C. Lizama, V. Poblete. On multiplicative perturbation of integral resolvent families, J. Math. Anal. Appl., 327 (2) (2007), 1335-1359.

[19] G.M. N'Guérékata. Existence and Uniqueness of Almost Automorphic Mild Solutions of Some Semilinear Abstract Differential Equations, Semigroup Forum 69 (2004), 80-86.

[20] G.M. N'Guérékata. Topics in almost automorphy, Springer Verlag, New York, 2005.

[21] G.M. N'Guerekata. Almost Automorphic and Almost Periodic Functions in Abstract Spaces, Kluwer Acad/Plenum, New York-Boston-Moscow-London, 2001.

[22] J.W. Nunziato. On heat conduction in materials with memory, Quart. Appl. Math. 29 (1971), 187-204.

[23] J. Prüss. Evolutionary Integral Equations and Applications. Monographs Math., 87, Birkhäuser Verlag, 1993.

[24] S.Y.Shaw, J.C. Chen. Asymptotic behavior of $(a, k)$-regularized families at zero, Taiwanese J. Math. 10 (2) (2006), 531-542.

Universidade Federal de Pernambuco, Departamento de Matemática,

Av. Prof. Luiz Freire, S/N, Recife-PE, CEP. 50540-740, Brazil.

E-mail address: cch@dmat.ufpe.br

Departamento de Matemática, Facultad de Ciencias. Universidad de Santiago de Chile, Casilla 307-Correo 2, Santiago, Chile

E-mail address: carlos.lizama@usach.cl 